\title{
On a singular limit for the compressible rotating Euler system
}

\author{
Šárka Nečasová ${ }^{* \dagger} \quad$ Tong Tang ${ }^{2,1 \ddagger \S}$ \\ 1. Institute of Mathematics of the Academy of Sciences of the Czech Republic, \\ Žitná 25, 11567, Praha 1, Czech Republic \\ 2. Department of Mathematics, College of Sciences, \\ Hohai University, Nanjing 210098, P.R. China
}

\begin{abstract}
The work addresses a singular limit for a rotating compressible Euler system in the low Mach number and low Rossby number regime. Based on the concept of dissipative measurevalued solution, the quasi-geostrophic system is identified as the limit problem in the case of ill-prepared initial data. The ill-prepared initial data will cause rapidly oscillating acoustic waves. Using dispersive estimates of Strichartz type, the effect of the acoustic waves in the asymptotic limit is eliminated.
\end{abstract}

Key words: compressible Euler equations, singular limit, low Mach number, low Rossby number, dissipative measure-valued solutions.

2010 Mathematics Subject Classifications: 35Q30.

\section{Introduction}

Earth's graceful rotation is an unignorable factor at geophysical fluids models. These models play an important role in the analysis of complex Earth phenomena in meteorology, geophysical and astrophysics. In order to describe the effect of rotation, people introduce two factors: Coriolis acceleration and centrifugal acceleration. In many real world applications, the action of centrifugal force is neglected, as it is in equilibrium with stratification caused by the gravity of the Earth. Under the above assumptions, we consider the following scaled Euler equations in an infinite slap $\Omega=\mathbb{R}^{2} \times(0,1)$ :

$$
\left\{\begin{array}{l}
\partial_{t} \rho+\operatorname{div}(\rho \mathbf{u})=0 \\
\partial_{t}(\rho \mathbf{u})+\operatorname{div}(\rho \mathbf{u} \otimes \mathbf{u})+\frac{1}{M a^{2}} \nabla_{x} p(\rho)+\frac{1}{R o} \rho(\omega \times \mathbf{u})=0
\end{array}\right.
$$

where the unknown fields $\rho=\rho(t, x)$ and $\mathbf{u}=\mathbf{u}(t, x)$ represent the density and the velocity of an inviscid compressible fluid, $\omega=(0,0,1)$ is the rotation axis. The Mach number Ma, proportional

\footnotetext{
*The research of Š.N. leading to these results has received funding from the Czech Sciences Foundation (GAČR), P201-16-032308 and RVO 67985840. Final version of the paper was made under support the Czech Sciences Foundation (GAČR), GA19-04243S.

†Email: matus@math.cas.cz

¥The research of T.T. is supported by the NSFC Grant No. 11801138 .

§Email: tt0507010156@126.com
} 
to the characteristic velocity field divided by the sound speed, and the Rossby number Ro, defined as the ratio of the displacement due to Coriolis forces, play the role of singular (small) parameters. The symbol $p=p(\rho)$ denotes the barotropic pressure (assumptions on the pressure see (3.1)). The system is supplemented by the far field conditions

$$
\mathbf{u} \rightarrow 0, \quad \rho \rightarrow \bar{\rho}, \text { as }|x| \rightarrow \infty, \text { where } \bar{\rho}>0
$$

and boundary condition

$$
\left.\mathbf{u} \cdot \mathbf{n}\right|_{\partial \Omega}=0
$$

where $\mathbf{n}$ is outer normal vector to $\partial \Omega$.

From modeling of geophysical fluids, the value of Mach number and Rossby number can be considered very small. It is well known that the compressible fluid flow becomes incompressible in the low Mach number limit, as the density distribution is constant and the velocity field becomes solenoidal. On the other hand, low Rossby number corresponds to fast rotation and the fast rotating fluids will lead to the so-called Taylor-Proudman columns phenomena. Therefore, it is interesting to observe the phenomenon if the two effects take place simultaneously. In this paper, we address the problem of the double limit for $M a=R o=\epsilon$. Let $\rho=\rho_{\epsilon}, \mathbf{u}=\mathbf{u}_{\epsilon}$, the system (1.1) takes the form

$$
\left\{\begin{array}{l}
\partial_{t} \rho_{\epsilon}+\operatorname{div}\left(\rho_{\epsilon} \mathbf{u}_{\epsilon}\right)=0 \\
\partial_{t}\left(\rho_{\epsilon} \mathbf{u}_{\epsilon}\right)+\operatorname{div}\left(\rho_{\epsilon} \mathbf{u}_{\epsilon} \otimes \mathbf{u}_{\epsilon}\right)+\frac{1}{\epsilon^{2}} \nabla_{x} p\left(\rho_{\epsilon}\right)+\frac{1}{\epsilon} \rho_{\epsilon}\left(\omega \times \mathbf{u}_{\epsilon}\right)=0
\end{array}\right.
$$

Our goal is to study the singular limit $\epsilon \rightarrow 0$ at the case of the ill-prepared initial data for the scaled system (1.4). The definition of ill-prepared initial data will be introduced in Section 3.3 . Supposing we know that in the corresponding spaces,

$$
\rho_{\epsilon}^{(1)}=\frac{\rho_{\epsilon}-\bar{\rho}}{\epsilon} \rightarrow q, \quad \mathbf{u}_{\epsilon} \rightarrow \mathbf{v}
$$

we can find that $q$ and $\mathbf{v}$ satisfy the following equations:

$$
\begin{aligned}
& \omega \times \mathbf{v}+\frac{p^{\prime}(\bar{\rho})}{\bar{\rho}} \nabla_{x} q=0, \\
& \partial_{t}\left(\Delta_{h} q-\frac{1}{p^{\prime}(\bar{\rho})} q\right)+\nabla_{h}^{\perp} q \cdot \nabla_{h}\left(\Delta_{h} q\right)=0 .
\end{aligned}
$$

Equations (1.5) (1.6) can be interpreted as a kind of stream function, according to physicists, named as quasi-physical flows 32 . For non-rotating compressible Euler fluids, a great number of well-posedness results have been obtained. However, some classical literatures show that smooth solutions of the Euler system will exhibit blow-up phenomena in a finite time no matter how smooth or small the initial data are. Therefore, it seems more appropriate to consider a suitable class of admissible weak solutions to (1.4). By admissible we mean that solutions will satisfy some form of the energy balance. The need for global admissible solutions of the Euler system leads to the concept of more general dissipative measure-valued (DMV) solutions introduced in the context of the full Euler system in [3, 4].

The measure-valued solutions to hyperbolic conservations laws were introduced by DiPerna [10. He used Young measures to pass to the artificial viscosity limit. In the case of the incompressible Euler equations, DiPerna and Majda [11] proved the global existence of measure-valued 
solutions for any initial data with finite energy. They introduced generalized Young measures to take into account oscillations and concentrations. Further, the existences of measure-valued solutions were shown for further models of fluids, e.g. compressible Euler and Navier-Stokes equations [26, 23. The measure-valued solution to the non-Newtonian case was proved by Novotný and Nečasová [25. The generalization was given by Alibert and Bouchitté [1. The weak-strong uniqueness for generalized measure-valued solutions of isentropic Newtonian Euler equations were proved in 21. Inspired by previous results, the concept of dissipative measure-valued solution was finally applied to the barotropic compressible Navier-Stokes system [19].

The reader may consult [17, 18, 23, 24, 26] for applications of the theory of (DMV) solutions in fluid mechanics or their counterparts [8, 9] in other areas of mathematical physics.

Let us discuss the main differences between weak solutions and (DMV) solutions. First important advanatge of $(D M V)$ solution is that DMV solutions to the compressible Euler system exist globally in time. Secondly $(D M V)$ solutions convergence to the limit system holds for any ill-prepared initial data, which in both case are not valid for weak solutions.

Due to the above fascinate advantage, there are some new results concerning singular limits in the context of measure-valued solutions. The low Mach number limit was studied in [18, where it is shown that $(D M V)$ solutions approach the smooth solutions of incompressible Euler system both for well-prepared and ill-prepared data. Moreover, the singular limit of compressible Euler system in the low Mach number and strong stratification regime for the ill-prepared data was identified, see [20]. However, to the best of our knowledge, compared with non-rotating case, there is a few results concerning on the singular limit of rotating compressible Euler system no matter weak solutions or strong solutions. Nilasis 28 , proved the singular limit of a rotating compressible Euler system with stratification at the case of well-prepared initial data. Our goal is to consider the asymptotic limit of $(D M V)$ solutions to the compressible Euler equations with ill-prepared initial data. We prove it converges to the strong solutions of quasi-physical flows. Moreover, we should emphasize that boundary conditions in this paper can be replaced by the periodical conditions in $x_{3}$ direction with a few changes. All the choices of boundary conditions prevent the flow from creating a viscous boundary layer. The periodic domain with well-prepared initial data was considered by Feireisl et al., [18. If the whole domain is torus $T^{3}$, it is difficult to obtain the analysis of acoustic waves at the case of ill-prepared initial data. It seems interesting to compare the results of the present paper with those obtained in [18. The analysis in [18] leans that the $(D M V)$ solutions of Euler system will converge to incompressible Euler system. Moreover, there is obvious difference about acoustic wave analysis between rotating and non-rotating case. The extension of the results of [18] to the rotating Euler system is therefore not straightforward. Last but not least, we should emphasis that there are huge results about rotating Navier-Stokes system such as [5, 6, 14, 15, 16.

The paper is organized as follows. In Section 2, we introduce the dissipative measure solutions, relative energy and the other necessary material. In Section 3, we state our main theorem. Section 4 is devoted to deriving uniform bounds of the Euler system independent of $\epsilon$. In Section 5, we perform the necessary analysis of the acoustic waves. The proof of the main theorem is completed in Section 6. 


\section{Preliminaries}

First let us observe that it is more convenient to rewrite the Euler system in terms of the conservative variables $\rho, \mathbf{m}=\rho \mathbf{u}$. Let $\mathcal{Q}=\left\{[\rho, \mathbf{m}] \mid \rho \in[0, \infty), \mathbf{m} \in \mathbb{R}^{3}\right\}$ be the natural phase space associated to solutions $[\rho, \mathbf{m}]=[\rho, \rho \mathbf{u}]$.

\subsection{Dissipative measure--valued solutions}

A dissipative measure-valued (DMV) solution of the Euler system (1.1) is a parameterized family of probability measures

$$
\left\{Y_{t, x}\right\}_{t \in[0, T], x \in \Omega}, \quad(t, x) \mapsto Y_{t, x} \in L_{\text {weak-(*) }}^{\infty}((0, T) \times \Omega ; \mathcal{P}(\mathcal{Q})),
$$

satisfying

- the continuity equation

$$
\int_{0}^{T} \int_{\Omega}\left[\left\langle Y_{t, x} ; \rho\right\rangle \partial_{t} \varphi+\left\langle Y_{t, x} ; \mathbf{m}\right\rangle \nabla_{x} \varphi\right] d x d t=-\int_{\Omega}\left\langle Y_{0, x} ; \rho\right\rangle \varphi(0) d x
$$

for all $\varphi \in C_{c}^{\infty}([0, T) \times \Omega)$;

- the momentum equation

$$
\begin{array}{r}
\int_{0}^{T} \int_{\Omega}\left[\left\langle Y_{t, x} ; \mathbf{m}\right\rangle \partial_{t} \varphi+\left\langle Y_{t, x} ; \frac{\mathbf{m} \otimes \mathbf{m}}{\rho}\right\rangle: \nabla_{x} \varphi\right] d x d t+\int_{0}^{T} \int_{\Omega}\left\langle Y_{t, x} ; p(\rho)\right\rangle \operatorname{div} \varphi d x d t \\
+\int_{0}^{T} \int_{\Omega}\left\langle Y_{t, x} ; \omega \times \mathbf{m}\right\rangle \varphi d x d t=-\int_{\Omega}\left\langle Y_{0, x} ; \mathbf{m}\right\rangle \varphi(0) d x-\int_{0}^{T} \int_{\Omega} \nabla_{x} \varphi: d \mu_{c}
\end{array}
$$

for all $\varphi \in C_{c}^{\infty}([0, T) \times \Omega)$, where $\mu_{c} \in \mathcal{M}([0, T] \times \Omega)$ is the so-called momentum concentration measure;

- the energy inequality

$$
\begin{array}{r}
\int_{\Omega}\left[\left\langle Y_{\tau, x} ; \frac{1}{2} \frac{|\mathbf{m}|^{2}}{\rho}+\left(P(\rho)-P^{\prime}(\bar{\rho})(\rho-\bar{\rho})-P(\bar{\rho})\right)\right\rangle d x+\mathcal{D}(\tau)\right. \\
\leq \int_{\Omega}\left\langle Y_{0, x} ; \frac{1}{2} \frac{|\mathbf{m}|^{2}}{\rho}+\left(P(\rho)-P^{\prime}(\bar{\rho})(\rho-\bar{\rho})-P(\bar{\rho})\right)\right\rangle d x,
\end{array}
$$

for a.a $\tau \in(0, T)$, where

$$
P(\rho)=\rho \int_{\bar{\rho}}^{\rho} \frac{p(z)}{z^{2}} d z
$$

and $\mathcal{D}$ is a non-negative function $\mathcal{D} \in L^{\infty}(0, T)$, satisfying the compatibility condition

$$
\int_{0}^{\tau} \int_{\Omega}\left|\mu_{c}\right| d x d t \leq C \int_{0}^{\tau} \xi(t) \mathcal{D}(t) d t, \text { for some } \xi \in L^{1}(0, T) .
$$

Remark 2.1. The notion of (DMV) solutions can be founded in many works as it was already mention in the Introduction, see e.g. [3, 4, 8, 9, 19]. For convenience of readers, we give more details.

Let $L_{\text {weak }}^{\infty}((0, T) \times \Omega ; P(Q))$ be the space of essentially bounded weakly ${ }^{\star}-$ measure maps $Y:(0, T) \times \Omega \rightarrow P(Q),(t, x) \mapsto Y_{t, x}$. By virtue of fundamental theorem on Young measures (see 
[2]) there exists a subsequence of $\left\{\rho_{\epsilon}, \mathbf{m}_{\epsilon}\right\}_{\epsilon>0}$ and parameterized family of probability measures $\left\{Y_{t, x}\right\}_{(t, x) \in(0, T) \times \Omega}$

$$
\left[(t, x) \mapsto Y_{t, x}\right] \in L_{w e a k \star}^{\infty}((0, T) \times \Omega ; P(Q)),
$$

such that a.a. $(t, x) \in(0, T) \times \Omega$

$$
\left\langle Y_{t, x} ; G(\rho, \mathbf{m})\right\rangle=\overline{G(\rho, \mathbf{m})}(t, x) \text { forany } G \in C_{c}(Q),
$$

whenever

$$
G\left(\rho_{\epsilon}, \mathbf{m}_{\epsilon}\right) \rightarrow \overline{G(\rho, \mathbf{m})}(t, x) \text { weakly } \star \text { in } L^{\infty}((0, T) \times \Omega) .
$$

Moreover, if $G \in C(Q)$ is such that

$$
\int_{0}^{T} \int_{\Omega}\left|G\left(\rho_{\epsilon}, \mathbf{m}_{\epsilon}\right)\right| d x \leq C,
$$

then $G$ is $Y_{t, x}$ integrable for almost all $(t, x) \in(0, T) \times \Omega$ and

$$
\left[(t, x) \mapsto\left\langle Y_{t, x} ; G(\rho, \mathbf{m})\right\rangle\right] \in L^{1}((0, T) \times \Omega),
$$

and

$$
G\left(\rho_{\epsilon}, \mathbf{m}_{\epsilon}\right) \rightarrow \overline{G(\rho, \mathbf{m})}(t, x) \text { weakly } \star \text { in } \mathcal{M}((0, T) \times \Omega) .
$$

The difference

$$
\mu_{G} \equiv \overline{G(\rho, \mathbf{m})}-\left[(t, x) \mapsto\left\langle Y_{t, x} ; G(\rho, \mathbf{m})\right\rangle\right] \in \mathcal{M}((0, T) \times \Omega),
$$

is called concentration defect measure.

For more details, please see [7].

Remark 2.2. $\quad$ - The measure $Y_{0, x}$ plays the role of initial conditions.

- The proof of an existence of (DMV) solutions of Euler system was done in the pioneer work by Neustupa, [26]. Recently, see [27], the authors proved the local strong solutions of rotating compressible Euler system in $\mathbb{R}^{3}$. Feireisl et al. [3, 4] proved the existence of (DMV) solutions to the non-rotating full Euler system. As the rotating term does not bring any trouble in the proof of existence, the existence of (DMV) solutions to (1.4) can be obtained by analogous methods as in [4].

Remark 2.3. We need to define the function

$$
[\rho, \mathbf{m}] \mapsto \frac{|\mathbf{m}|^{2}}{\rho}
$$

on the vacuum set as

$$
[\rho, \mathbf{m}] \rightarrow \frac{|\mathbf{m}|^{2}}{\rho}=\left\{\begin{array}{l}
\infty, \text { if } \rho=0 \text { and } \mathbf{m} \neq 0 \\
\frac{|\mathbf{m}|^{2}}{\rho}, \text { if } \rho>0, \\
0, \text { otherwise. }
\end{array}\right.
$$

Accordingly, it follows from the energy inequality (2.4) that

$$
\left.\operatorname{Supp}\left[Y_{t, x}\right] \cap\{[\rho, \mathbf{m}] \in \mathcal{Q} \mid \rho=0, \mathbf{m} \neq 0]\right\}=\emptyset \text { for a.a. }(t, x) \text {. }
$$




\subsection{Relative entropy inequality}

Motivated by [12, 13, 4, we introduce the relative energy functional

$$
\mathcal{E}(\rho, \mathbf{m} \mid r, \mathbf{U})=\int_{\Omega}\left\langle Y_{t, x} ; \frac{1}{2} \rho\left|\frac{\mathbf{m}}{\rho}-\mathbf{U}(t, x)\right|^{2}+\left(P(\rho)-P^{\prime}(r)(\rho-r)-P(r)\right)\right\rangle d x,
$$

where $r>0$, $\mathbf{U}$ are smooth "test" functions, $r-\bar{\rho}, U$ compactly supported in $\Omega$.

As shown in [4], any (DMV) solution of (1.1) satisfies the relative entropy inequality

$$
\begin{aligned}
\mathcal{E} & \left.(\rho, \mathbf{m} \mid r, \mathbf{U})\right|_{t=0} ^{t=\tau}+\mathcal{D}(\tau) \leq \int_{0}^{\tau} \int_{\Omega}\left\langle Y_{t, x} ;\left(\partial_{t} \mathbf{U}+\frac{\mathbf{m}}{\rho} \nabla_{x} U\right)(\rho \mathbf{U}-\mathbf{m})\right\rangle d x d t \\
& +\int_{0}^{\tau} \int_{\Omega}\left\langle Y_{t, x} ;(r-\rho) \partial_{t} P^{\prime}(r)+(r \mathbf{U}-\mathbf{m}) \nabla_{x} P^{\prime}(r)\right\rangle d x d t+\int_{0}^{\tau} \int_{\Omega}\left\langle Y_{t, x} ; \omega \times \frac{\mathbf{m}}{\rho}\right\rangle(\rho \mathbf{U}-\mathbf{m}) d x d t \\
& -\int_{0}^{\tau} \int_{\Omega}\left\langle Y_{t, x} ; p(\rho)-p(r)\right\rangle \operatorname{div} \mathbf{U} d x d t+\int_{0}^{\tau} \int_{\Omega} \nabla_{x} \mathbf{U}: d \mu_{c} .
\end{aligned}
$$

for a.a. $\tau \in[0, T]$, and any $r, \mathbf{U} \in C^{1}([0, T] \times \Omega), r-\bar{\rho}$, $\mathbf{U}$ compactly supported in $\Omega$.

\section{Main result}

Before stating our main result, we introduce some notations and collect several mostly technical hypotheses and known facts concerning the limit system. $x=\left(x_{h}, x_{3}\right)$ with $x_{h} \in \mathbb{R}^{2}$ denoting its horizontal component. For a vector field $\mathbf{b}=\left[b_{1}, b_{2}, b_{3}\right]$, we introduce the horizontal component $\mathbf{b}_{h}=\left[b_{1}, b_{2}\right]$ writing $\mathbf{b}=\left[\mathbf{b}_{h}, b_{3}\right]$. Similarly, we use the symbols $\nabla_{h}$, div $\operatorname{div}_{h}$ to denote the differential operators acting on the horizontal variables. The following assumptions and results will be used in the proof.

\section{$3.1 \quad$ Pressure}

We suppose the pressure $p$ is a continuously differentiable function of the density such that for some $\gamma>1$,

$$
p \in C^{1}[0, \infty) \cap C^{\infty}(0, \infty), p(0)=0, p^{\prime}(\rho)>0 \text { for all } \rho>0, \lim _{\rho \rightarrow \infty} \frac{p^{\prime}(\rho)}{\rho^{\gamma-1}}=p_{\infty}>0 .
$$

Remark 3.1. Similarly to [18], we deduce that

$$
\begin{aligned}
& p(\rho)-p^{\prime}(r)(\rho-r)-p(r) \text { is dominated by } P(\rho)-P^{\prime}(r)(\rho-r)-P(r), \text { specifically, } \\
& |\rho-r|^{2} \leq c(\delta)\left(P(\rho)-P^{\prime}(r)(\rho-r)-P(r)\right) \quad \text { when } 0<\delta \leq \rho, r \leq \frac{1}{\delta}, \delta>0, \\
& 1+|\rho-r|+P(\rho) \leq c(\delta)\left(P(\rho)-P^{\prime}(r)(\rho-r)-P(r)\right) \quad \text { if } 0<2 \delta<r<\frac{1}{2 \delta}, \\
& \rho \in[0, \delta) \cup\left(\frac{1}{\delta}, \infty\right), \delta>0 .
\end{aligned}
$$

\subsection{Quasi-geophysical equation}

The expected limit problem reads

$$
\omega \times \mathbf{v}+\frac{p^{\prime}(\bar{\rho})}{\bar{\rho}} \nabla_{x} q=0, \mathbf{v}=\left[\mathbf{v}_{\mathbf{h}}\left(x_{h}\right), 0\right], q=q\left(x_{h}\right),
$$




$$
\partial_{t}\left(\Delta_{h} q-\frac{1}{p^{\prime}(\bar{\rho})} q\right)+\mathbf{v}_{h} \cdot \nabla_{h}\left(\Delta_{h} q\right)=0 .
$$

supplement with the initial condition

$$
\left.q\right|_{t=0}=q_{0} .
$$

As shown by Oliver [29, the problem (3.2) - (3.3) possesses a unique classical solution

$$
q \in C\left([0, T] ; W^{m, 2}\left(\mathbb{R}^{2}\right)\right) \cap C^{1}\left([0, T] ; W^{m-1,2}\left(\mathbb{R}^{2}\right)\right), \quad m \geq 4,
$$

for any initial solution

$$
q_{0} \in W^{m, 2}\left(\mathbb{R}^{2}\right)
$$

\subsection{Ill prepared initial-data}

The ill-prepared initial data for the scaled system (1.4) take the form

$$
\rho_{\epsilon}(0, \cdot)=\rho_{0, \epsilon}=\bar{\rho}+\epsilon s_{0, \epsilon}, \mathbf{u}_{\epsilon}(0, \cdot)=\mathbf{u}_{0, \epsilon}
$$

where

$$
\begin{gathered}
s_{0, \epsilon} \rightarrow s_{0} \text { in } W^{k, 2}(\Omega) \cap W^{k, 1}(\Omega), \quad \mathbf{u}_{0, \epsilon} \rightarrow \mathbf{u}_{0} \text { in } W^{k, 2}(\Omega) \cap W^{k, 1}(\Omega), \quad(k>3), \\
\mathbf{u}_{0}=\mathbf{v}_{0}+\nabla_{x} \Phi_{0} .
\end{gathered}
$$

\subsection{Singular limit - main result}

For simplicity, we assume $\bar{\rho}=p^{\prime}(\bar{\rho})=P^{\prime \prime}(\bar{\rho})=1$. Now, we are ready to state our main result.

Theorem 3.1. Let $\left\{Y_{t, x}^{\epsilon}\right\}_{(t, x) \in[0, T] \times \Omega}$ be a family of $(D M V)$ solutions to the scaled Euler system (1.4) satisfying the compatibility condition (2.6) with a function $\xi$ independent of $\epsilon$. Let the initial data $\left\{Y_{0, x}^{\epsilon}\right\}_{x \in \Omega}$ be ill-prepared, namely

$$
\int_{\Omega}\left\langle Y_{0, x}^{\epsilon} ; \frac{1}{2} \rho\left|\frac{\mathbf{m}}{\rho}-\mathbf{u}_{0, \epsilon}(x)\right|^{2}+\frac{1}{\epsilon^{2}}\left(P(\rho)-P^{\prime}\left(\rho_{0, \epsilon}\right)\left(\rho-\rho_{0, \epsilon}\right)-P\left(\rho_{0, \epsilon}\right)\right)\right\rangle d x \rightarrow 0,
$$

where $\rho_{0, \epsilon}, \mathbf{u}_{0, \epsilon}$ are ill prepared data introduced in Section 3.3

Then

$$
\begin{aligned}
& \mathcal{D}^{\epsilon} \rightarrow 0 \text { in } L^{\infty}(0, T), \\
& Y_{t, x}^{\epsilon} \rightarrow \delta_{[q, \mathbf{v}]} \text { in } L^{p}\left(0, T ; L_{\mathrm{loc}}^{1}\left(\Omega ; \mathcal{M}^{+}(\mathcal{Q})_{\text {weak }-(\star)}\right)\right) \text { for any finite } p \geq 1,
\end{aligned}
$$

where $q$ and $\mathbf{v}$ is the unique solution of problem (3.2)-(3.3) starting from the initial data $q_{0}$ and where $q_{0} \in W^{k+1,2}\left(\mathbb{R}^{2}\right) \cap W^{k+1,1}\left(\mathbb{R}^{2}\right)$ is the unique solution of the elliptic problem

$$
-\Delta_{h} q_{0}+q_{0}=\int_{0}^{1} \operatorname{curl}_{h}\left[\mathbf{u}_{0}\right]_{h} d x_{3}+\int_{0}^{1} s_{0} d x_{3} .
$$

The rest of the paper is devoted to the proof of Theorem 3.1. 


\section{Energy bounds}

We start by deriving uniform bounds on solutions to (1.4) independent of $\epsilon$. Similarly to [18], we introduce the decomposition

$$
h(\rho, \mathbf{m})=[h]_{\text {ess }}(\rho, \mathbf{m})+[h]_{\text {res }}(\rho, \mathbf{m}), \quad[h]_{\text {ess }}=\psi(\rho) h(\rho, \mathbf{m}), \quad[h]_{\text {res }}=(1-\psi(\rho)) h(\rho, \mathbf{m}),
$$

where

$$
\psi \in C_{c}^{\infty}(0, \infty), \quad 0 \leq \psi(\rho) \leq 1, \quad \psi(\rho)=1 \text { on an open interval containing } \bar{\rho}=1
$$

As the initial data are ill-prepared, the expression on the right-hand side of the energy inequality (2.4) remains bounded uniformly for $\epsilon \rightarrow 0$. Consequently, we deduce the following bound:

$$
\text { ess } \sup _{t \in(0, T)} \int_{\Omega}\left\langle Y_{t, x}^{\epsilon} ; \frac{1}{2} \frac{\left|\mathbf{m}_{\epsilon}\right|^{2}}{\rho}+\frac{1}{\epsilon^{2}}\left(P\left(\rho_{\epsilon}\right)-P^{\prime}(1)\left(\rho_{\epsilon}-1\right)-P(1)\right)\right\rangle d x \leq C .
$$

Thus, exactly as in [18, we use the structural properties of the function $p$ to deduce

$$
\begin{aligned}
& \text { ess } \sup _{t \in(0, T)} \int_{\Omega}\left\langle Y_{t, x}^{\epsilon} ;\left|\left[\frac{\rho_{\epsilon}-1}{\epsilon}\right]_{e s s}\right|^{2}\right\rangle+\left\langle Y_{t, x}^{\epsilon} ;\left[\frac{P\left(\rho_{\epsilon}\right)+1}{\epsilon^{2}}\right]_{e s s}\right\rangle d x \leq C ; \\
& (t, x) \mapsto\left\langle Y_{t, x}^{\epsilon} ; \mathbf{m}_{\epsilon}\right\rangle \text { bounded in } L^{\infty}\left(0, T ; L^{2}(\Omega)+L^{\frac{2 \gamma}{\gamma+1}}(\Omega)\right) ; \\
& (t, x) \mapsto\left\langle Y_{t, x}^{\epsilon} ;\left[\frac{\rho_{\epsilon}-1}{\epsilon}\right]_{e s s}\right\rangle \text { bounded in } L^{\infty}\left(0, T ; L^{2}(\Omega)\right) ; \\
& (t, x) \mapsto \epsilon^{-\frac{2}{\gamma}}\left\langle Y_{t, x}^{\epsilon} ;\left[\rho_{\epsilon}\right]_{r e s}\right\rangle \text { bounded in } L^{\infty}\left(0, T ; L^{\gamma}(\Omega)\right) .
\end{aligned}
$$

Using the same argument in [17, there exist functions $\rho^{(1)} \in L^{\infty}\left(0, T ; L^{2}(\Omega)\right)$ and $\mathbf{m} \in$ $L^{\infty}\left(0, T ; L^{q}(\Omega)\right)$ for some $q>1$ and a subsequence such that

$$
\begin{aligned}
& \left\langle Y_{t, x}^{\epsilon} ; \mathbf{m}_{\epsilon}\right\rangle \rightarrow \mathbf{m} \text { weakly in } L^{\infty}\left(0, T ; L^{q}(\Omega)\right. \\
& \left\langle Y_{t, x}^{\epsilon} ; \frac{\rho_{\epsilon}-1}{\epsilon}\right\rangle \rightarrow \rho^{(1)} \text { weakly in } L^{\infty}\left(0, T ; L^{2}(\Omega)\right) .
\end{aligned}
$$

Recalling (2.2) and (2.3), we deduce

$$
\int_{0}^{T} \int_{\Omega} \mathbf{m} \cdot \nabla_{x} \varphi d x d t=0, \quad \int_{0}^{T} \int_{\Omega}\left[(\omega \times \mathbf{m}) \cdot \varphi+\rho^{(1)} \operatorname{div} \varphi\right] d x d t=0,
$$

for $\varphi \in C^{1}([0, T] \times \Omega)$. In other words,

$$
\operatorname{div}_{x} \mathbf{m}=0, \omega \times \mathbf{m}+\nabla_{x} \rho^{(1)}=0
$$

in the sense of distribution.

It is easy to check that

$$
\rho^{(1)}=\rho^{(1)}\left(x_{h}\right), \mathbf{m}=\left(\mathbf{m}_{h}, 0\right), \operatorname{div}_{x} \mathbf{m}=\operatorname{div}_{h} \mathbf{m}_{h}=0 .
$$

Moreover, the detail of derivation of (3.9) can be seen in [14, 15, 16]. 


\section{Acoustic waves}

It is well-known that ill-prepared data give rise to rapidly oscillating acoustic waves. Similarly to [15, the relevant acoustic equation reads

$$
\left\{\begin{array}{l}
\epsilon \partial_{t} s_{\epsilon}+\operatorname{div}\left(\nabla_{x} \Phi_{\epsilon}\right)=0 \\
\epsilon \partial_{t} \nabla_{x} \Phi_{\epsilon}+\omega \times \nabla_{x} \Phi_{\epsilon}+\nabla_{x} s_{\epsilon}=0
\end{array}\right.
$$

supplemented with the initial data

$$
s_{\epsilon}(0, \cdot)=s_{0}, \nabla_{x} \Phi_{\epsilon}(0, \cdot)=\nabla_{x} \Phi_{0},
$$

where $s_{0}, \nabla_{x} \Phi_{0}$ have been introduced in Section 3.3 .

As a matter of fact, the initial data must be smoothed and cut-off via suitable regularization operators, namely

$$
s_{\epsilon}(0, \cdot)=s_{0, \delta}=\left[s_{0}\right]_{\delta} ; \quad \nabla_{x} \Phi_{\epsilon}(0, \cdot)=\nabla_{x} \Phi_{0, \delta}=\nabla_{x}\left[\Phi_{0}\right]_{\delta},
$$

where $[\cdot]_{\delta}$ denotes the regularization introduced in [15].

Denoting the corresponding solutions $s_{\epsilon, \delta}, \Phi_{\epsilon, \delta}$ we report the following energy and dispersive estimates proved in [15, Section 6]:

$$
\sup _{t \in[0, T]}\left[\left\|\Phi_{\epsilon, \delta}(t, \cdot)\right\|_{W^{m, 2}}+\left\|s_{\epsilon, \delta}(t, \cdot)\right\|_{W^{m, 2}}\right]=\left[\left\|\nabla_{x} \Phi_{0, \delta}\right\|_{L^{2}}+\left\|s_{0, \delta}\right\|_{L^{2}}\right],
$$

and

$$
\int_{0}^{T}\left[\left\|\Phi_{\epsilon, \delta}(t, \cdot)\right\|_{W^{m, \infty}}+\left\|s_{\epsilon, \delta}(t, \cdot)\right\|_{W^{m, \infty}}\right] \leq \omega(\epsilon, m, \delta)\left[\left\|\nabla_{x} \Phi_{0, \delta}\right\|_{L^{2}}+\left\|r_{0, \delta}\right\|_{L^{2}}\right],
$$

where $\omega(\epsilon, m, \delta) \rightarrow 0$ as $\epsilon \rightarrow 0$ for any fixed $m \geq 0$ and $\delta>0$. More details about Strichartz estimates and acoustic waves, readers can refer to [30, 31.

\section{Convergence}

The proof of convergence is based on the ansatz

$$
r_{\epsilon}=1+\epsilon\left(q+s_{\epsilon, \delta}\right), \mathbf{U}_{\epsilon}=\mathbf{v}+\nabla_{x} \Phi_{\epsilon, \delta},
$$

in the relative energy inequality (2.10). The $\left[s_{\epsilon, \delta}, \nabla_{x} \Phi_{\epsilon, \delta}\right]$ are solutions of the acoustic system (5.1), and $[q, \mathbf{v}]$ is solution of the target problem

$$
\begin{aligned}
& \omega \times \mathbf{v}+\nabla_{x} q=0, \\
& \partial_{t}\left(\Delta_{h} q-q\right)+\nabla_{h}^{\perp} q \cdot \nabla_{h}\left(\Delta_{h} q\right)=0 .
\end{aligned}
$$

In addition, to avoid technicalities, we shall assume that $s_{0}$ and $\Phi_{0}$ are sufficiently regular so that the $\delta$-regularization is not needed in (5.1-5.3). Accordingly, we have $s_{\epsilon, \delta}=s_{\epsilon}, \Phi_{\epsilon, \delta}=\Phi_{\epsilon}$. The general case may be handled as in [15].

First note that the relative energy for the scaled system reads

$$
\mathcal{E}_{\epsilon}\left(\rho_{\epsilon}, \mathbf{m}_{\epsilon} \mid r_{\epsilon}, \mathbf{U}_{\epsilon}\right)=\int_{\Omega}\left\langle Y_{t, x} ; \frac{1}{2} \rho_{\epsilon}\left|\frac{\mathbf{m}_{\epsilon}}{\rho_{\epsilon}}-\mathbf{U}_{\epsilon}\right|^{2}+\frac{1}{\epsilon^{2}}\left(P\left(\rho_{\epsilon}\right)-P^{\prime}\left(r_{\epsilon}\right)\left(\rho_{\epsilon}-r_{\epsilon}\right)-P\left(r_{\epsilon}\right)\right)\right\rangle d x,
$$


with the corresponding relative energy inequality:

$$
\begin{aligned}
& \left.\mathcal{E}_{\epsilon}\left(\rho_{\epsilon}, \mathbf{m}_{\epsilon} \mid r_{\epsilon}, \mathbf{U}_{\epsilon}\right)\right|_{t=0} ^{t=\tau}+\mathcal{D}^{\epsilon}(\tau) \leq \int_{0}^{\tau} \int_{\Omega}\left\langle Y_{t, x}^{\epsilon} ; \rho_{\epsilon} \mathbf{U}_{\epsilon}-\mathbf{m}_{\epsilon}\right\rangle\left(\partial_{t} \mathbf{U}_{\epsilon}+\frac{\mathbf{m}_{\epsilon}}{\rho_{\epsilon}} \nabla_{x} \mathbf{U}_{\epsilon}\right) d x d t \\
& \quad+\frac{1}{\epsilon^{2}} \int_{0}^{\tau} \int_{\Omega}\left[\left\langle Y_{t, x}^{\epsilon} ; r_{\epsilon}-\rho_{\epsilon}\right\rangle \partial_{t} P^{\prime}\left(r_{\epsilon}\right)+\left\langle Y_{t, x}^{\epsilon} ; r_{\epsilon} \mathbf{U}_{\epsilon}-\mathbf{m}_{\epsilon}\right\rangle \nabla_{x} P^{\prime}\left(r_{\epsilon}\right)\right] d x d t \\
& \quad+\frac{1}{\epsilon} \int_{0}^{\tau} \int_{\Omega}\left\langle Y_{t, x} ; \omega \times \frac{\mathbf{m}_{\epsilon}}{\rho_{\epsilon}}\right\rangle\left(\rho_{\epsilon} \mathbf{U}_{\epsilon}-\mathbf{m}_{\epsilon}\right) d x d t-\frac{1}{\epsilon^{2}} \int_{0}^{\tau} \int_{\Omega}\left\langle Y_{t, x} ; p\left(\rho_{\epsilon}\right)-p\left(r_{\epsilon}\right)\right\rangle \operatorname{div} \mathbf{U}_{\epsilon} d x d t \\
& \quad+\int_{0}^{\tau} \int_{\Omega} \nabla_{x} \mathbf{U}_{\epsilon}: d \mu_{c} .
\end{aligned}
$$

Our goal is to show that, with the ansatz (6.1), the relative energy $\mathcal{E}_{\epsilon}\left(\rho_{\epsilon}, \mathbf{m}_{\epsilon} \mid r_{\epsilon}, \mathbf{U}_{\epsilon}\right)$ tends to zero for $\epsilon \rightarrow 0$ uniformly in $t \in[0, T]$. In view of the dispersive estimates $(5.2)-(5.3)$, this will yield the conclusion claimed in Theorem 3.1. To this end, we use a Gronwall type argument showing that all integrals in the right-hand side of (6.4) are either small or can be absorbed by the left-hand side as $\epsilon \rightarrow 0$. This programme will be carried over by means of several steps.

\subsection{Step 1}

First, we compute

$$
\begin{aligned}
& \int_{0}^{\tau} \int_{\Omega}\left[\left\langle Y_{t, x}^{\epsilon} ; r_{\epsilon}-\rho_{\epsilon}\right\rangle \partial_{t} P^{\prime}\left(r_{\epsilon}\right)+\left\langle Y_{t, x}^{\epsilon} ; r_{\epsilon} \mathbf{U}_{\epsilon}-\mathbf{m}_{\epsilon}\right\rangle \nabla_{x} P^{\prime}\left(r_{\epsilon}\right)-\left\langle Y_{t, x}^{\epsilon} ; p\left(\rho_{\epsilon}\right)-p\left(r_{\epsilon}\right)\right\rangle \operatorname{div} \mathbf{U}_{\epsilon}\right] d x d t \\
& =\int_{0}^{\tau} \int_{\Omega}\left[\left\langle Y_{t, x}^{\epsilon} ; p\left(r_{\epsilon}\right)-p^{\prime}\left(r_{\epsilon}\right)\left(r_{\epsilon}-\rho_{\epsilon}\right)-p\left(\rho_{\epsilon}\right)\right\rangle \operatorname{div} \mathbf{U}_{\epsilon}+\left\langle Y_{t, x}^{\epsilon} ; r_{\epsilon}-\rho_{\epsilon}\right\rangle \partial_{t} P^{\prime}\left(r_{\epsilon}\right)\right. \\
& \left.\quad+\left\langle Y_{t, x}^{\epsilon} ;\left(r_{\epsilon}-\rho_{\epsilon}\right) p^{\prime}\left(r_{\epsilon}\right)\right\rangle \operatorname{div} \mathbf{U}_{\epsilon}+\left\langle Y_{t, x}^{\epsilon} ;\left(r_{\epsilon}-\rho_{\epsilon}\right) \nabla_{x} P^{\prime}\left(r_{\epsilon}\right)\right\rangle \mathbf{U}_{\epsilon}+\left\langle Y_{t, x}^{\epsilon} ;\left(\rho_{\epsilon} \mathbf{U}_{\epsilon}-\mathbf{m}_{\epsilon}\right) \nabla_{x} P^{\prime}\left(r_{\epsilon}\right)\right\rangle\right] d x d t \\
& =\int_{0}^{\tau} \int_{\Omega}\left[\left\langle Y_{t, x}^{\epsilon} ; p\left(r_{\epsilon}\right)-p^{\prime}\left(r_{\epsilon}\right)\left(r_{\epsilon}-\rho_{\epsilon}\right)-p\left(\rho_{\epsilon}\right)\right\rangle \operatorname{div} \mathbf{U}_{\epsilon}+\left\langle Y_{t, x}^{\epsilon} ; \partial_{t} r_{\epsilon}+\operatorname{div}_{x}\left(r_{\epsilon} \mathbf{U}_{\epsilon}\right)\right\rangle\left(r_{\epsilon}-\rho_{\epsilon}\right) P^{\prime \prime}\left(r_{\epsilon}\right)\right. \\
& \left.\quad+\left\langle Y_{t, x}^{\epsilon} ;\left(\rho_{\epsilon} \mathbf{U}_{\epsilon}-\mathbf{m}_{\epsilon}\right) \nabla_{x} P^{\prime}\left(r_{\epsilon}\right)\right\rangle\right] d x d t .
\end{aligned}
$$

Note that, in view of (6.2),

$$
\begin{aligned}
\partial_{t} r_{\epsilon}+\operatorname{div}_{x}\left(r_{\epsilon} \mathbf{U}_{\epsilon}\right) & =\epsilon \partial_{t} q+\partial_{t} s_{\epsilon}+\operatorname{div}\left(r_{\epsilon}\left(\mathbf{v}+\nabla_{x} \Phi_{\epsilon}\right)\right) \\
& \left.=\epsilon \partial_{t} q+\epsilon \operatorname{div}\left(\left(q+s_{\epsilon}\right) \mathbf{U}_{\epsilon}\right)\right) .
\end{aligned}
$$

Next, by virtue of (5.1) and (6.1),

$$
\begin{aligned}
\nabla_{x} P^{\prime}\left(r_{\epsilon}\right)\left(\rho_{\epsilon} \mathbf{U}_{\epsilon}-\mathbf{m}_{\epsilon}\right)= & \nabla_{x}\left(P^{\prime}\left(r_{\epsilon}\right)-P^{\prime \prime}(1)\left(r_{\epsilon}-1\right)-P^{\prime}(1)\right)\left(\rho_{\epsilon} \mathbf{U}_{\epsilon}-\mathbf{m}_{\epsilon}\right)+\epsilon \nabla_{x} q \cdot\left(\rho_{\epsilon} \mathbf{U}_{\epsilon}-\mathbf{m}_{\epsilon}\right) \\
& +\epsilon \nabla_{x} s_{\epsilon} \cdot\left(\rho_{\epsilon} \mathbf{U}_{\epsilon}-\mathbf{m}_{\epsilon}\right) \\
= & \nabla_{x}\left(P^{\prime}\left(r_{\epsilon}\right)-P^{\prime \prime}(1)\left(r_{\epsilon}-1\right)-P^{\prime}(1)\right)\left(\rho_{\epsilon} \mathbf{U}_{\epsilon}-\mathbf{m}_{\epsilon}\right)+\epsilon \nabla_{x} q \cdot\left(\rho_{\epsilon} \mathbf{U}_{\epsilon}-\mathbf{m}_{\epsilon}\right) \\
& \quad-\epsilon^{2}\left(\rho_{\epsilon} \mathbf{U}_{\epsilon}-\mathbf{m}_{\epsilon}\right) \cdot \partial_{t} \nabla \Phi_{\epsilon}-\epsilon\left(\rho_{\epsilon} \mathbf{U}_{\epsilon}-\mathbf{m}_{\epsilon}\right)\left(\omega \times \nabla \Phi_{\epsilon}\right) .
\end{aligned}
$$

Furthermore, by virtue of the compatibility condition (2.6), we can control the concentration measure,

$$
\int_{0}^{\tau} \int_{\Omega} \nabla_{x} \mathbf{U}: d \mu_{c} \leq\left\|\nabla_{x} \mathbf{U}\right\|_{L^{\infty}} \int_{0}^{\tau} \xi(t) \mathcal{D}^{\epsilon}(t) d t .
$$


Finally, as the hypotheses about the ill-prepared initial data, we have

$$
\mathcal{E}_{\epsilon}\left(\rho_{\epsilon}, \mathbf{m}_{\epsilon} \mid r_{\epsilon}, \mathbf{U}_{\epsilon}\right)(0) \rightarrow 0 \text { as } \epsilon \rightarrow 0 .
$$

Thus we may conclude that

$$
\begin{aligned}
& \mathcal{E}_{\epsilon}\left(\rho_{\epsilon}, \mathbf{m}_{\epsilon} \mid r_{\epsilon}, \mathbf{U}_{\epsilon}\right)(\tau)+\mathcal{D}^{\epsilon}(\tau) \leq \int_{0}^{\tau} \int_{\Omega}\left\langle Y_{t, x}^{\epsilon} ;\left(\partial_{t} \mathbf{v}+\frac{\mathbf{m}_{\epsilon}}{\rho_{\epsilon}} \nabla_{x} \mathbf{U}_{\epsilon}\right)\left(\rho_{\epsilon} \mathbf{U}_{\epsilon}-\mathbf{m}_{\epsilon}\right)\right\rangle d x d t \\
& \quad+\frac{1}{\epsilon} \int_{0}^{\tau} \int_{\Omega}\left\langle Y_{t, x}^{\epsilon} ; \omega \times \mathbf{v}\right\rangle\left(\rho_{\epsilon} \mathbf{U}_{\epsilon}-\mathbf{m}_{\epsilon}\right) d x d t \\
& \quad+\frac{1}{\epsilon^{2}} \int_{0}^{\tau} \int_{\Omega}\left\langle Y_{t, x}^{\epsilon} ; \nabla_{x}\left(P^{\prime}\left(r_{\epsilon}\right)-P^{\prime \prime}(1)\left(r_{\epsilon}-1\right)-P^{\prime}(1)\right)\right\rangle\left(\rho_{\epsilon} \mathbf{U}_{\epsilon}-\mathbf{m}_{\epsilon}\right) d x d t \\
& \quad-\frac{1}{\epsilon^{2}} \int_{0}^{\tau} \int_{\Omega}\left\langle Y_{t, x}^{\epsilon} ; p\left(\rho_{\epsilon}\right)-p\left(r_{\epsilon}\right)-p^{\prime}\left(r_{\epsilon}\right)\left(\rho_{\epsilon}-r_{\epsilon}\right)\right\rangle \operatorname{div} \mathbf{U}_{\epsilon} d x d t \\
& \left.\quad+\frac{1}{\epsilon} \int_{0}^{\tau} \int_{\Omega}\left\langle Y_{t, x}^{\epsilon} ; \partial_{t} q+\operatorname{div}\left(\left(q+s_{\epsilon}\right) \mathbf{U}_{\epsilon}\right)\right)\right\rangle\left(r_{\epsilon}-\rho_{\epsilon}\right) P^{\prime \prime}\left(r_{\epsilon}\right) d x d t \\
& \quad+\frac{1}{\epsilon} \int_{0}^{\tau} \int_{\Omega}\left\langle Y_{t, x}^{\epsilon} ; \nabla_{x} q\right\rangle\left(\rho_{\epsilon} \mathbf{U}_{\epsilon}-\mathbf{m}_{\epsilon}\right) d x d t+c \int_{0}^{\tau} \xi(t) \mathcal{D}^{\epsilon}(t) d t+\omega(\epsilon),
\end{aligned}
$$

where $\omega(\epsilon)$ denotes a generic quantity satisfying

$$
\omega(\epsilon) \rightarrow 0 \text { in } L^{1}(0, T) \text { as } \epsilon \rightarrow 0 .
$$

Using (6.2), we get the following conclusion:

$$
\begin{aligned}
\mathcal{E}_{\epsilon}\left(\rho_{\epsilon}, \mathbf{m}_{\epsilon} \mid r_{\epsilon}, \mathbf{U}_{\epsilon}\right)(\tau)+\mathcal{D}^{\epsilon}(\tau) \leq \int_{0}^{\tau} \int_{\Omega}\left\langle Y_{t, x}^{\epsilon} ;\left(\partial_{t} \mathbf{v}+\frac{\mathbf{m}_{\epsilon}}{\rho_{\epsilon}} \nabla_{x} \mathbf{U}_{\epsilon}\right)\left(\rho_{\epsilon} \mathbf{U}_{\epsilon}-\mathbf{m}_{\epsilon}\right)\right\rangle d x d t \\
\quad+\frac{1}{\epsilon^{2}} \int_{0}^{\tau} \int_{\Omega}\left\langle Y_{t, x}^{\epsilon} ; \nabla_{x}\left(P^{\prime}\left(r_{\epsilon}\right)-P^{\prime \prime}(1)\left(r_{\epsilon}-1\right)-P^{\prime}(1)\right)\right\rangle\left(\rho_{\epsilon} \mathbf{U}_{\epsilon}-\mathbf{m}_{\epsilon}\right) d x d t \\
\quad-\frac{1}{\epsilon^{2}} \int_{0}^{\tau} \int_{\Omega}\left\langle Y_{t, x}^{\epsilon} ; p\left(\rho_{\epsilon}\right)-p\left(r_{\epsilon}\right)-p^{\prime}\left(r_{\epsilon}\right)\left(\rho_{\epsilon}-r_{\epsilon}\right)\right\rangle \operatorname{div} \mathbf{U}_{\epsilon} d x d t \\
\left.\quad+\frac{1}{\epsilon} \int_{0}^{\tau} \int_{\Omega}\left\langle Y_{t, x}^{\epsilon} ; \partial_{t} q+\operatorname{div}\left(\left(q+s_{\epsilon}\right) \mathbf{U}_{\epsilon}\right)\right)\right\rangle\left(r_{\epsilon}-\rho_{\epsilon}\right) P^{\prime \prime}\left(r_{\epsilon}\right) d x d t+c \int_{0}^{\tau} \xi(t) \mathcal{D}^{\epsilon}(t) d t+\omega(\epsilon),
\end{aligned}
$$

\subsection{Step 2}

We write

$$
\begin{aligned}
\int_{0}^{\tau} \int_{\Omega}[ & \left.\left\langle\mathbf{Y}_{t, x}^{\epsilon} ; \rho_{\epsilon} U_{\epsilon}-\mathbf{m}_{\epsilon}\right\rangle\left(\partial_{t} \mathbf{v}+\frac{\mathbf{m}_{\epsilon}}{\rho_{\epsilon}} \nabla_{x} \mathbf{U}_{\epsilon}\right)\right] d x d t \\
= & \int_{0}^{\tau} \int_{\Omega}\left\langle Y_{t, x}^{\epsilon} ; \rho_{\epsilon} \mathbf{U}_{\epsilon}-\mathbf{m}_{\epsilon}\right\rangle\left(\partial_{t} \mathbf{v}+\mathbf{v} \cdot \nabla_{x} \mathbf{v}\right) d x d t \\
& +\int_{0}^{\tau} \int_{\Omega}\left\langle Y_{t, x}^{\epsilon} ; \rho_{\epsilon} \mathbf{U}_{\epsilon}-\mathbf{m}_{\epsilon}\right\rangle\left(\mathbf{v} \cdot \nabla_{x} \nabla_{x} \Phi_{\epsilon}+\nabla_{x} \Phi_{\epsilon} \nabla_{x} \mathbf{U}_{\epsilon}\right) d x d t \\
& +\int_{0}^{\tau} \int_{\Omega}\left\langle Y_{t, x}^{\epsilon} ; \rho_{\epsilon} \mathbf{U}_{\epsilon}-\mathbf{m}_{\epsilon}\right\rangle\left(\frac{\mathbf{m}_{\epsilon}}{\rho_{\epsilon}}-\mathbf{U}_{\epsilon}\right) \nabla_{x} \mathbf{U}_{\epsilon} d x d t \\
= & I_{1}+I_{2}+I_{3} .
\end{aligned}
$$


Using the uniform bounds (4.2), we can split the functions in $I_{2}$ into their essential and residual parts obtaining

$$
\begin{aligned}
& \left|\int_{\mathbb{R}^{2}}\left\langle Y_{t, x}^{\epsilon} ; \rho_{\epsilon} \mathbf{U}_{\epsilon}-\mathbf{m}_{\epsilon}\right\rangle\left(\mathbf{v} \cdot \nabla_{x} \nabla_{x} \Phi_{\epsilon}+\nabla_{x} \Phi_{\epsilon} \nabla_{x} \mathbf{U}_{\epsilon}\right) d x\right| \\
& \leq\left\|\nabla_{x} \Phi_{\epsilon}\right\|_{W^{1, \infty}}^{2}\left(\|\mathbf{v}\|_{W^{3,2}}+\left\|\nabla_{x} \mathbf{U}_{\epsilon}\right\|_{W^{3,2}}\right)^{2}+c \mathcal{E}_{\epsilon}\left(\rho_{\epsilon}, \mathbf{m}_{\epsilon} \mid r_{\epsilon}, \mathbf{U}_{\epsilon}\right),
\end{aligned}
$$

where the first term on the right-hand side can be controlled by means of the dispersive estimate (5.2) and (5.3).

Summing up the previous observations, we may infer that the relative energy inequality with the ansatz (6.1) reduces to

$$
\begin{aligned}
\mathcal{E}_{\epsilon}\left(\rho_{\epsilon}, \mathbf{m}_{\epsilon} \mid r_{\epsilon}, \mathbf{U}_{\epsilon}\right)(\tau)+\mathcal{D}^{\epsilon}(\tau) \leq \int_{0}^{\tau} \int_{\Omega}\left\langle Y_{t, x}^{\epsilon} ; \rho_{\epsilon} \mathbf{U}_{\epsilon}-\mathbf{m}_{\epsilon}\right\rangle\left(\partial_{t} \mathbf{v}+\mathbf{v} \cdot \nabla_{x} \mathbf{v}\right) d x d t \\
+\frac{1}{\epsilon^{2}} \int_{0}^{\tau} \int_{\Omega}\left\langle Y_{t, x}^{\epsilon} ; \nabla_{x}\left(P^{\prime}\left(r_{\epsilon}\right)-P^{\prime \prime}(1)\left(r_{\epsilon}-1\right)-P^{\prime}(1)\right)\right\rangle\left(\rho_{\epsilon} \mathbf{U}_{\epsilon}-\mathbf{m}_{\epsilon}\right) d x d t \\
-\frac{1}{\epsilon^{2}} \int_{0}^{\tau} \int_{\Omega}\left\langle Y_{t, x}^{\epsilon} ; p\left(\rho_{\epsilon}\right)-p\left(r_{\epsilon}\right)-p^{\prime}\left(r_{\epsilon}\right)\left(\rho_{\epsilon}-r_{\epsilon}\right)\right\rangle \operatorname{div} \mathbf{U}_{\epsilon} d x d t \\
\quad-\frac{1}{\epsilon} \int_{0}^{\tau} \int_{\Omega}\left\langle Y_{t, x}^{\epsilon} ;\left(\rho_{\epsilon}-r_{\epsilon}\right) P^{\prime \prime}\left(r_{\epsilon}\right)\right\rangle\left(\partial_{t} q+\operatorname{div}\left(\left(q+s_{\epsilon}\right) \mathbf{U}_{\epsilon}\right)\right) d x d t \\
+C \int_{0}^{\tau} \mathcal{E}_{\epsilon}\left(\rho_{\epsilon}, \mathbf{m}_{\epsilon} \mid r_{\epsilon}, \mathbf{U}_{\epsilon}\right) d t+C \int_{0}^{\tau} \xi(t) \mathcal{D}^{\epsilon}(t) d t+\omega(\epsilon) .
\end{aligned}
$$

\subsection{Step 3}

Now, we will deal with pressure term and corresponding term. First, using direct calculation, the Taylor formula and dispersive estimates (5.2-5.3), we deduce that

$$
\begin{aligned}
\frac{1}{\epsilon^{2}} \mid \nabla_{x}\left(P^{\prime}\left(r_{\epsilon}\right)-\right. & \left.P^{\prime}(1)-P^{\prime \prime}(1)\left(r_{\epsilon}-1\right)\right) \mid \\
& =\frac{1}{\epsilon}\left|\left(P^{\prime \prime}\left(r_{\epsilon}\right)-P^{\prime \prime}(1)\right) \nabla_{x}\left(q+s_{\epsilon}\right)\right| \\
& \rightarrow P^{\prime \prime \prime}(1) q \nabla_{x} q \text { as } \epsilon \rightarrow 0 .
\end{aligned}
$$

Therefore, combining the previous energy bounds and convergence, we get

$$
\frac{1}{\epsilon^{2}} \int_{0}^{\tau}\left\langle Y_{t, x}^{\epsilon} ; \nabla_{x}\left(P^{\prime}\left(r_{\epsilon}\right)-P^{\prime}(1)-P^{\prime \prime}(1)\left(r_{\epsilon}-1\right)\right)\right\rangle\left(\rho_{\epsilon} \mathbf{U}_{\epsilon}-\mathbf{m}_{\epsilon}\right) d t \rightarrow 0 \text { as } \epsilon \rightarrow 0 .
$$

The remaining pressure term is

$$
\begin{aligned}
& \left|\frac{1}{\epsilon^{2}} \int_{0}^{\tau}\left\langle Y_{t, x}^{\epsilon} ; p\left(\rho_{\epsilon}\right)-p\left(r_{\epsilon}\right)-p^{\prime}\left(r_{\epsilon}\right)\left(\rho_{\epsilon}-r_{\epsilon}\right)\right\rangle \operatorname{div} \mathbf{U}_{\epsilon} d t\right| \\
& \quad=\left|\frac{1}{\epsilon^{2}} \int_{0}^{\tau}\left\langle Y_{t, x}^{\epsilon} ; p\left(\rho_{\epsilon}\right)-p\left(r_{\epsilon}\right)-p^{\prime}\left(r_{\epsilon}\right)\left(\rho_{\epsilon}-r_{\epsilon}\right)\right\rangle\left(\operatorname{div} \mathbf{v}+\Delta \Phi_{\epsilon}\right) d t\right| \\
& \quad \leq c\left|\frac{1}{\epsilon^{2}} \int_{0}^{\tau}\left\langle Y_{t, x}^{\epsilon} ; P\left(\rho_{\epsilon}\right)-P\left(r_{\epsilon}\right)-P^{\prime}\left(r_{\epsilon}\right)\left(\rho_{\epsilon}-r_{\epsilon}\right)\right\rangle\left(\operatorname{div} \mathbf{v}+\Delta \Phi_{\epsilon}\right) d t\right| \\
& \quad \leq C \int_{0}^{\tau} \mathcal{E}_{\epsilon}\left(\rho_{\epsilon}, \mathbf{m}_{\epsilon} \mid r_{\epsilon}, \mathbf{U}_{\epsilon}\right) d t,
\end{aligned}
$$


where we have used the previous dispersive estimates (5.2) and (5.3). Thus, we can conclude that

$$
\begin{aligned}
& \mathcal{E}_{\epsilon}\left(\rho_{\epsilon}, \mathbf{m}_{\epsilon} \mid r_{\epsilon}, \mathbf{U}_{\epsilon}\right)(\tau)+\mathcal{D}^{\epsilon}(\tau) \leq \omega(\epsilon)+C \int_{0}^{\tau} \mathcal{E}\left(\rho_{\epsilon}, \mathbf{m}_{\epsilon} \mid r_{\epsilon}, U_{\epsilon}\right) d t+c \int_{0}^{\tau} \xi(t) \mathcal{D}^{\epsilon}(t) d t \\
& \quad+\int_{0}^{\tau} \int_{\Omega}\left\langle Y_{t, x}^{\epsilon} ; \rho_{\epsilon} \mathbf{U}_{\epsilon}-\mathbf{m}_{\epsilon}\right\rangle\left(\partial_{t} \mathbf{v}+\mathbf{v} \cdot \nabla_{x} \mathbf{v}\right) d x d t \\
& \quad-\frac{1}{\epsilon} \int_{0}^{\tau} \int_{\Omega}\left\langle Y_{t, x}^{\epsilon} ;\left(\rho_{\epsilon}-r_{\epsilon}\right) P^{\prime \prime}\left(r_{\epsilon}\right)\right\rangle\left(\partial_{t} q+\operatorname{div}\left(\left(q+s_{\epsilon}\right) \mathbf{U}_{\epsilon}\right)\right) d x d t .
\end{aligned}
$$

\subsection{Step 4}

Finally, we deal with the remaining pressure terms. Similar to the previous analysis, we obtain

$$
\begin{aligned}
& \mathcal{E}_{\epsilon}\left(\rho_{\epsilon}, \mathbf{m}_{\epsilon} \mid r_{\epsilon}, \mathbf{U}_{\epsilon}\right)(\tau)+\mathcal{D}^{\epsilon}(\tau) \leq \omega(\epsilon)+C \int_{0}^{\tau} \mathcal{E}\left(\rho_{\epsilon}, \mathbf{m}_{\epsilon} \mid r_{\epsilon}, U_{\epsilon}\right) d t+c \int_{0}^{\tau} \xi(t) \mathcal{D}^{\epsilon}(t) d t \\
& \quad+\int_{0}^{\tau} \int_{\Omega}\left\langle Y_{t, x}^{\epsilon} ; \mathbf{v}-\mathbf{m}\right\rangle\left(\partial_{t} \mathbf{v}+\mathbf{v} \cdot \nabla_{x} \mathbf{v}\right) d x d t+\int_{0}^{\tau} \int_{\Omega}\left\langle Y_{t, x}^{\epsilon} ; q-\rho^{(1)}\right\rangle\left(\partial_{t} q+\operatorname{div}(q \mathbf{v})\right) d x d t,
\end{aligned}
$$

where

$$
\begin{aligned}
& \int_{0}^{\tau} \int_{\Omega}\left\langle Y_{t, x}^{\epsilon} ; \mathbf{v}-\mathbf{m}\right\rangle\left(\partial_{t} \mathbf{v}+\mathbf{v} \cdot \nabla_{x} \mathbf{v}\right) d x d t+\int_{0}^{\tau} \int_{\Omega}\left\langle Y_{t, x}^{\epsilon} ; q-\rho^{(1)}\right\rangle\left(\partial_{t} q+\operatorname{div}(q \mathbf{v})\right) d x d t \\
& =\frac{1}{2} \int_{0}^{\tau} \int_{\Omega}\left\langle Y_{t, x}^{\epsilon} ; \partial_{t}|\mathbf{v}|^{2}+\partial_{t}|q|^{2}\right\rangle d x d t-\int_{0}^{\tau} \int_{\Omega}\left\langle Y_{t, x}^{\epsilon} ; \partial_{t} \mathbf{v} \cdot \mathbf{m}+\partial_{t} q \cdot \rho^{(1)}\right\rangle d x d t \\
& \quad-\int_{0}^{\tau} \int_{\Omega}\left\langle Y_{t, x}^{\epsilon} ; \mathbf{v} \cdot \nabla_{x} \mathbf{v} \cdot \mathbf{m}+\rho^{(1)} \operatorname{div}(q \mathbf{v})\right\rangle d x d t
\end{aligned}
$$

By virtue of (4.3) and (6.2), we have

$$
\operatorname{div}_{x}(q \mathbf{v})=\nabla_{x} q \cdot \mathbf{v}=\nabla_{x} q \cdot \nabla_{x}^{\perp} q=0
$$

and

$$
-\int_{0}^{\tau} \int_{\Omega}\left\langle Y_{t, x}^{\epsilon} ; \partial_{t} \mathbf{v} \cdot \mathbf{m}+\partial_{t} q \rho^{(1)}\right\rangle d x d t=-\int_{0}^{\tau} \int_{\Omega}\left\langle Y_{t, x}^{\epsilon} ; \omega \times \mathbf{m}\right\rangle \mathbf{v} \Delta_{h} q d x d t
$$

Moreover, it is easy to check that

$$
\mathbf{v} \cdot \nabla_{x} \mathbf{v} \cdot \mathbf{m}+(\omega \times \mathbf{m}) \cdot \mathbf{v} \Delta_{h} q=\mathbf{m} \cdot \nabla_{h} \frac{|\mathbf{v}|^{2}}{2} .
$$

So we deduce that

$$
\begin{aligned}
\frac{1}{2} \int_{0}^{\tau} \int_{\Omega}\left\langle Y_{t, x}^{\epsilon} ; \partial_{t}|\mathbf{v}|^{2}+\partial_{t}|q|^{2}\right\rangle d x d t & =\frac{1}{2} \int_{0}^{\tau} \int_{\Omega}\left\langle Y_{t, x}^{\epsilon} ; \partial_{t}\left|\nabla_{h}^{\perp} q\right|^{2}+\partial_{t}|q|^{2}\right\rangle d x d t \\
& =\int_{0}^{\tau} \int_{\Omega}\left\langle Y_{t, x}^{\epsilon} ; \mathbf{v} \cdot \nabla_{h}\left(\Delta_{h} q\right) q\right\rangle d x d t \\
& =-\int_{0}^{\tau} \int_{\Omega}\left\langle Y_{t, x}^{\epsilon} ; \mathbf{v} \cdot \nabla_{h} q \Delta_{h} q\right\rangle d x d t=0,
\end{aligned}
$$

where the last equality due to $\mathbf{v}=\nabla^{\perp} q$. 
Putting together Step 1 to Step 4, we conclude that

$$
\mathcal{E}_{\epsilon}\left(\rho_{\epsilon}, \mathbf{m}_{\epsilon} \mid r_{\epsilon}, \mathbf{U}_{\epsilon}\right)+\mathcal{D}^{\epsilon}(\tau) \leq \omega(\epsilon)+\int_{0}^{\tau}(1+\xi(t))\left[\mathcal{E}\left(\rho_{\epsilon}, \mathbf{m}_{\epsilon} \mid r_{\epsilon}, \mathbf{U}_{\epsilon}\right)+\mathcal{D}^{\epsilon}(t)\right] d t
$$

where $r_{\epsilon}, \mathbf{U}_{\epsilon}$ are given by (6.1). Letting $\epsilon \rightarrow 0$ and applying the Gronwall's lemma, we complete the proof of Theorem 3.1.

\section{Acknowledgements}

The authors are grateful to the referee and the editor whose comments and suggestions greatly improved the presentation of this paper. The paper was written when Tong Tang was visiting the Institute of Mathematics of the Czech Academy of Sciences which hospitality and support is gladly acknowledged.

\section{References}

[1] J. J. Alibert and G. Bouchitté, Non-uniform integrability and generalized Young measures, J. Convex Anal., 4 (1997), 129-147.

[2] J. M. Ball, A version of the fundamental theorem for Young measures. PDEs and continuum models of phase transitions (Nice, 1988), Lecture Notes in Phys., 344, Springer, Berlin, (1988), 207-215.

[3] J. Březina and E. Feireisl, Measure-valued solutions to the complete Euler system revisited, Z. Angew. Math. Phys., 69 (2018), Art. 57, 17 pp.

[4] J. Březina and E. Feireisl, Measure-valued solutions to the complete Euler system, J. Math. Soc. Japan, 70 (2018), 1227-1245.

[5] M. Caggio and Š. Nečasová, Inviscid incompressible limits for rotating fluids, Nonlinear Anal., 163 (2017), 1-18.

[6] J. -Y. Chemin, B. Desjardins, I. Gallagher and E. Grenier, An introduction to rotating fluids and the Navier-Stokes equations, Oxford University Press, Oxford, 2006.

[7] E. Chiodaroli, E. Feireisl, Eduard, O. Kreml, E. Wiedemann, A-free rigidity and applications to the compressible Euler system, Ann.Mat. Pura Appl. 196 (2017), 1557-1572.

[8] S. Demoulini, D. M. A. Stuart and A. E. Tzavaras, Weak-strong uniqueness of dissipative measure-valued solutions for polyconvex elastodynamics, Arch. Ration. Mech. Anal., 205 (2012), 927-961.

[9] C. M. Dafermos, The second law of thermodynamics and stability, Arch. Rational Mech. Anal., 70 (1979) 167-179.

[10] R. J. DiPerna, Measure-valued solutions to conservation laws, Arch. Rational Mech. Anal., 88 (1985), 223-270. 
[11] R. J. DiPerna and A. Majda, Oscillations and concentrations in weak solutions of the incompressible fluid equations, Comm. Math. Phys., 108 (1987), 667-689.

[12] E. Feireisl, J. B. Jin and A. Novotný, Relative entropies, suitable weak solutions, and weak-strong uniqueness for the compressible Navier-Stokes system, J. Math. Fluid Mech., 14 (2012), 717-730.

[13] E. Feireisl and A. Novotný, Singular limits in thermodynamics of viscous fluids, Advances in Mathematical Fluid Mechanics, Birkhäuser, Basel, 2009.

[14] E. Feireisl, I. Gallagher and A. Novotný, A singular limit for compressible rotating fluids, SIAM J. Math. Anal., 44 (2012), 192-205.

[15] E. Feireisl and A. Novotný, Multiple scales and singular limits for compressible rotating fluids with general initial data, Comm. Partial Differential Equations, 39 (2014), 1104-1127.

[16] E. Feireisl and A. Novotný, Scale interactions in compressible rotating fluids, Ann. Mat. Pura Appl., 193 (2014), 1703-1725.

[17] E. Feireisl, C. Klingenberg, O. Kreml and S. Markfelder, On oscillatory solutions to the complete Euler system, arXiv:1710.10918, 2017.

[18] E. Feireisl, C. Klingenberg and S. Markfelder, On the low Mach number limit for the compressible Euler system, SIAM Journal on Mathematical Analysis, 51 (2019), 1496-1513.

[19] E. Feireisl, P. Gwiazda, A. Swierczewska-Gwiazda and E. Wiedemann, Dissipative measurevalued solutions to the compressible Navier-Stokes system, Calc. Var. Partial Differential Equations, 55 (2016), Art. 141, 20 pp.

[20] E. Feireisl and T. Tang, On a singular limit for the stratified compressible Euler system, Asymptot. Anal., 114 (2019), 59-72.

[21] P. Gwiazda, A. Swierczewska-Gwiazda and E. Wiedemann, Weak-strong uniqueness for measure-valued solutions of some compressible fluid models, Nonlinearity, 28 (2015), 38733890.

[22] S. Klainerman and A. Majda, Singular limits of quasilinear hyperbolic systems with large parameters and the incompressible limit of compressible fluids, Comm. Pure Appl. Math., 34 (1981), 481-524.

[23] D. Kröner and W. Zajaczkowski, Measure-valued solutions of the Euler equations for ideal compressible polytropic fluids, Math. Methods Appl. Sci., 19 (1996), 235-252.

[24] J. Málek, J. Nečas, M. Rokyta and M. Růžička, Weak and measure-valued solutions to evolutionary PDEs, Applied Mathematics and Mathematical Computation, London, 1996.

[25] Matušů - Nečasová Š. and A. Novotný, Measure-valued solution for non-Newtonian compressible isothermal monopolar fluid, Mathematical problems for Navier-Stokes equations (Centro, 1993), Acta Appl. Math., 37(1-2), 109-128, 1994. 
[26] J. Neustupa, Measure-valued solutions of the Euler and Navier-Stokes equations for compressible barotropic fluids, Math. Nachr., 163 (1993), 217-227.

[27] V. S. Ngo and S. Scrobogna, Dispersive effects of weakly compressible and fast rotating inviscid fluids, Discrete Contin. Dyn. Syst., 38 (2018), 749-789.

[28] N. Chaudhuri, Multiple scales and singular limits of perfect fluids, arxiv: 1909.08529v1.

[29] M. Oliver, Classical solutions for a generalized Euler equation in two dimensions, J. Math. Anal. Appl., 215 (1997), 471-484

[30] S. Schochet, The mathematical theory of low Mach number flows, M2AN Math. Model. Numer. Anal., 39 (2005), 441-458.

[31] H. F. Smith and C. D. Sogge, Global Strichartz estimates for nontrapping perturbations of the Laplacian, Comm. Partial Differential Equations, 25 (2000), 2171-2183.

[32] V. Zeitlin, Nonlinear Dynamics of Rotating Shallow Water, Methods and Advances, SpringerVerlag, Heidelberg, 2006. 